\title{
Apendicite aguda puerperal: Relato de caso
}

\section{Acute puerperal appendicitis: Case report}

DOI: $10.46814 / 1 a j d v 3 n 6-024$

Recebimento dos originais: 01/11/2021

Aceitação para publicação: 15/12/2021

\author{
Prof. Dr. Joe Luiz Vieira Garcia Novo \\ Professor Titular do Departamento de Reprodução Humana e Infância - Faculdade de Ciências \\ Médicas e da Saúde/PUC-SP. \\ Rua Manoel Ferreira Leão, 67 ap. 21 Vila Leão - CEP 18040-410 Sorocaba-SP \\ E-mail: joeluiznovo@ hotmail.com \\ Christiane Machado Chen \\ Ex-aluna do Curso de Medicina da Faculdade de Ciências Médicas e da Saúde/PUC-SP.
}

\section{RESUMO}

A apendicite aguda é a causa cirúrgica não obstétrica mais comum no ciclo grávido puerperal, incluindo as puérperas. $\mathrm{O}$ atraso no diagnóstico em virtude de a sua sintomatologia apresentar-se geralmente atenuada ou ausente, ou por ser confundida com alterações fisiológicas do puerpério, poderá ocasionar aumento de duas a três vezes a perfuração apendicular, peritonite puerperal, resultando em alguns casos em mortalidade materna. Relata-se um caso de apendicite aguda puerperal ulcero-necrotizante e a sua evolução pós-operatória. $\mathrm{O}$ aumento de duas a três vezes na presença de perfuração do apêndice e peritonite nas puérperas, podendo resultar grave morbidade e/ou mortalidade, ressaltam a importância da divulgação da frequência de casos de apendicite aguda em puérperas entre clínicos e obstetras. É de suma importância que estejam atentos em relação a sua ocorrência, e assim possam realizar o diagnóstico o mais precocemente possível, visando a redução do número de complicações e da mortalidade destas pacientes.

Palavra chaves: puerpério, abdômen agudo, apendicite aguda.

\begin{abstract}
Acute appendicitis is the most common non-obstetric surgical cause in the pregnant puerperal cycle, including puerperal women. The delay in diagnosis because its symptoms are generally attenuated or absent, or because it is confused with physiological changes in the puerperium, may cause a two to three-fold increase in appendicular perforation, puerperal peritonitis, resulting in some cases in maternal mortality. We report a case of ulcerative-necrotic puerperal acute appendicitis and its postoperative evolution. The two to three-fold increase in the presence of appendix perforation and peritonitis in postpartum women, which can result in severe morbidity and/or mortality, emphasizes the importance of disclosing the frequency of cases of acute appendicitis in postpartum women among clinicians and obstetricians. It is extremely important to be aware of its occurrence, and thus be able to make the diagnosis as early as possible, aiming to reduce the number of complications and mortality of these patients.
\end{abstract}

Keywords: puerperium, acute abdomen, acute appendicitis. 


\section{INTRODUÇÃO}

O abdômen agudo no ciclo grávido puerperal, em geral, é patologia rara. Náuseas, vômitos, dor abdominal presentes em gestações fisiológicas, além de leucocitose ao exame de hemograma, frequentemente úteis ao diagnóstico diferencial, podem dificultar e/ou confundir além do diagnóstico, as condutas adequadas a serem adotados. $1-5$

A apendicite aguda é a causa cirúrgica não obstétrica mais comum durante a gravidez 4, 5 Sua frequência na puérpera (1: 1000 gestações) é igual à da gestante. É patologia de fácil tratamento, porém, o atraso no diagnóstico em virtude de a sua sintomatologia apresentar-se geralmente atenuada ou ausente, ou por ser confundida com alterações fisiológicas do puerpério, poderá ocasionar aumento de duas a três vezes na ocorrência de perfuração apendicular, consequente peritonite puerperal, resultando em alguns casos na sua mortalidade. 6, $7 \mathrm{O}$ tratamento curativo desta patologia, é o procedimento cirúrgico. 8

Assinale-se que após o lançamento do Programa de Humanização no Pré-natal e Nascimento (PHPN), o mesmo, foi de grande utilidade como sendo instrumento de reconhecimento e de melhora na resolução das altas taxas de morbiletalidade materno-fetal que até então ocorriam, incluindo a patologia em epígrafe. 9 A assistência obstétrica obteve outrossim, reconhecimento dos recursos tecnológicos 10 e de colaboração governamental, constituindo maior eficiência da assistência obstétrica. 11

Em virtude das peculiaridades, dificuldades de diagnóstico e de prognóstico materno, que acompanham a ocorrência de apendicite aguda durante o período puerperal, o objetivo deste relato é lembrar e prevenir que "a mortalidade da apendicite aguda complicando a gravidez, é a mortalidade da demora". 12

\section{RELATO DE CASO}

N.P.R., 24 anos de idade, primigesta na $41^{\mathrm{a}}$ semana de gestação, foi admitida na maternidade do Hospital Santa Lucinda de Sorocaba-SP, para indução do trabalho de parto, indicada por pósdatismo. Ocorrendo falha de indução ao parto, após 14 horas, foi submetida a parto cesárea, realizado sem intercorrências, recém-nascido masculino, pesando 3.900g, boa vitalidade ao nascer. Ambos receberam alta hospitalar após 48 horas. Decorridos 15 dias após o parto, a puérpera retornou ao hospital por estar sentindo dores intensas na região da fossa ilíaca direita, que se irradiavam para toda a região abdominal, acompanhada de febre $\left(38^{\circ} \mathrm{C}\right)$ e vômito. Medicada com analgésicos, foi liberada para sua casa como tratamento inicial. Sem melhora, após 4 semanas foi internada para avaliação clínica, por apresentar abdome distendido, doloroso à palpação, e sinal de descompressão brusca positivo no flanco direito. Identificada leucocitose discreta inespecífica (11.460/ mm3), proteína C 
reativa elevada $(48.01 \mathrm{mg} / \mathrm{dL})$, ultrassonografia de abdome total descrevendo apêndice cecal espessado $(1,2 \mathrm{~cm})$, com conteúdo hipoecóico associado a hiperecogenicidade da gordura adjacente (achados compatíveis com processo inflamatório do apêndice), foi indicada a apendicectomia (fig. 1),

Fig.1 Apêndice cecal espessado $(1,2 \mathrm{~cm})$ com conteúdo hipoecóico associado a hiperecogenicidade da gordura adjacente, achados compatíveis com processo inflamatório do apêndice.

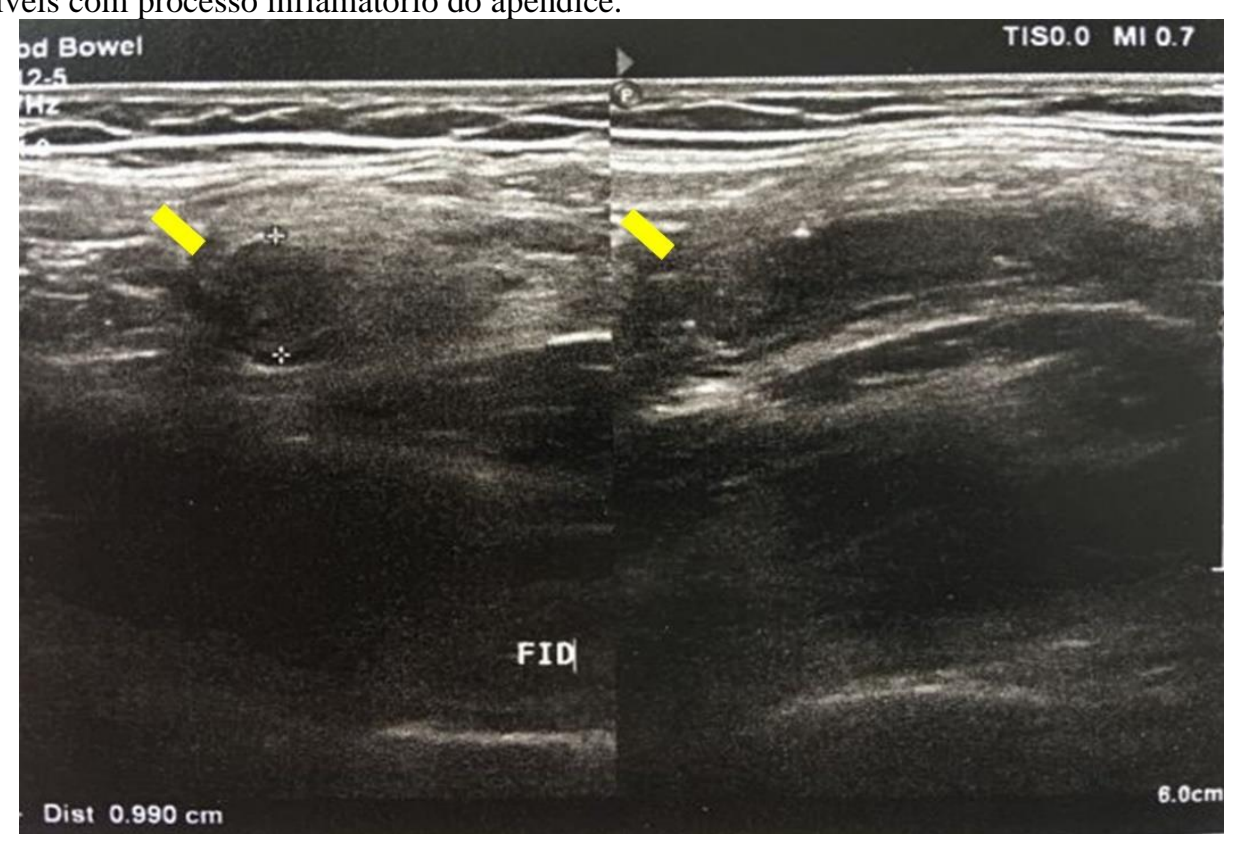

A cirurgia e o pós-operatório sucederam-se sem intercorrências. Dois dias após a cirurgia a paciente recebeu alta hospitalar. $\mathrm{O}$ apêndice cecal macroscopicamente mediu $5,3 \mathrm{~cm}$ de comprimento e $0,7 \mathrm{~cm}$ de diâmetro, serosa parcialmente recoberta por fibrina, mesoapêndice aderido a ela; a luz apresentava-se dilatada, preenchida por material pastoso e acastanhado. No exame microscópico observou-se extensa necrose parietal apendicular, associada a difusa exsudação de fibrina com neutrófilos íntegros e degenerados. O laudo anatomopatológico indicou o diagnóstico como apendicite aguda ulcero-necrotizante. Foi reinternada 20 dias após a alta cirúrgica, apresentando cicatrização parcial e a saída de secreção purulenta, com odor fétido pela incisão operatória. Novo exame ultrassonográfico revelou coleção heterogênea, de conteúdo espesso, predominantemente hipoecogênica, medindo aproximadamente 2,9 x 1,6 x 2,6 cm (volume estimado em 6,2cm3), associada a densificação dos planos gordurosos adjacentes em fossa ilíaca direita, com aparente pertuito para a pele na topografia da cicatriz cirúrgica (fig. 2). Sob tratamento antibioticoterápico específico, houve melhora do quadro clínico, recebeu alta 10 dias após, e encaminhada para acompanhamento em Unidade Básica de Saúde (UBS). 


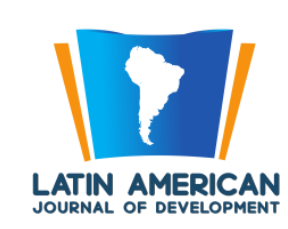

Fig.2 Coleção heterogênea, de conteúdo espesso, predominantemente hipoecogênica, medindo aproximadamente 2,9 x 1,6 x $2,6 \mathrm{~cm}$ (volume estimado em $6,2 \mathrm{~cm}^{3}$ ), associada a densificação dos planos gordurosos adjacentes em fossa ilíaca direita, com aparente pertuito para a pele na topografia da cicatriz cirúrgica.

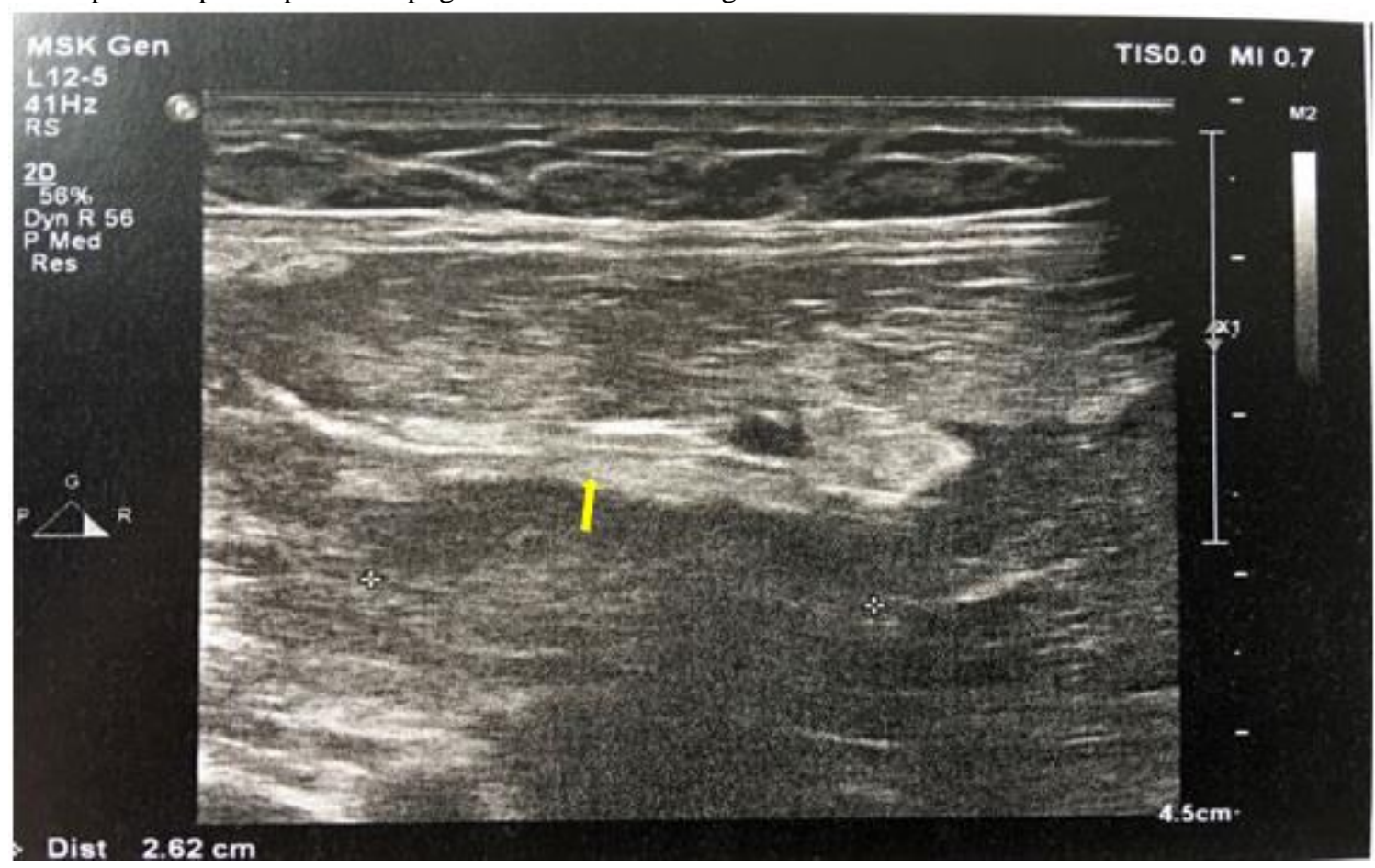

\section{DISCUSSÃO}

Durante o transcorrer do puerpério, o apêndice não retorna ao seu posicionamento anatômico habitual, até a completa involução uterina, a qual, em geral, se realiza em seis a oito semanas após o parto. Além disto, o útero aumentado tende a deslocar a parede abdominal, afastando-a do apêndice quando inflamado. Este deslocamento associado à hipotonia da musculatura da parede abdominal pósgestacional, poderá minimizar os sinais clínicos de irritação peritoneal, tornando as manifestações clínicas da apendicite aguda pouco evidentes. 6

As pacientes classicamente descrevem inicialmente a queixa dolorosa, com localização primária, em geral, ao redor da área periumbilical migrando posteriormente ao quadrante abdominal direito. Anorexia, náuseas ou vômitos surgem a seguir. Quadro febril e leucocitose poderão complementar a síndrome clínica. 13 - 15

Considere-se que a dor abdominal no puerpério tem multiplicidade de diferentes patologias (infecções pélvicas, gastroenterite, úlcera péptica, obstrução intestinal, hepatite, pancreatite, colecistite, apendicite, peri-hepatite, pneumonia, pielonefrite, etc.), e a abordagem inicial pode gerar dúvidas. 1, 2

O diagnóstico clínico de apendicite durante o ciclo grávido puerperal, em geral, é dificultado pelas características próprias das fases gestacionais, e, por conseguinte, são recomendados estudos de imagem, com o fito de minimizar atrasos na cirurgia, incertezas diagnósticas, e, além disto, reduzir taxas de apendicectomias negativas. 16 
A ultrassonografia total abdominal é o método de imagem inicial, para a provável solução diagnóstica, podendo afastar outras afecções, diagnosticar a apendicite aguda, localizar a posição do apêndice, facilitando o acesso cirúrgico. 2 A suspeita da patologia associa-se com a identificação de imagem tubular não compressível de extremidade cega, em geral, localizada no quadrante inferior direito, podendo até apresentar diâmetro superior a $6 \mathrm{~mm} .17,18$

A utilização da tomografia computadorizada tem sua utilidade diagnósica reconhecida. A ressonância magnética indisponível em muitos lugares de nosso país, contribui para a afirmação da apendicite aguda. 19.A certeza diagnóstica levará imediatamente para o tratamento cirúrgico, pois atrasos iguais ou superiores a 24 horas, aumentam o risco de perfuração. 2, 18

A abordagem laparoscópica ao invés de cirurgia abdominal aberta, vem adquirindo cada vez mais aceitação, graças aos resultados favoráveis de sua utilização, proporcionando mobilização precoce da paciente, além de rápida recuperação pós-operatória. 2, 6 Acresçam-se os benefícios da laparoscopia a ser utilizada em pacientes com dor abdominal inespecífica, associada à suspeição de apendicite aguda, incluem, também, elevação de acerto diagnóstico, além de diminuição de remoção de apêndices normas, quando se compara com a abertura cirúrgica abdominal. 20

Vimos que fatores limitantes relacionados à gravidez, podem complicar o diagnóstico de apendicite aguda. Estas pacientes necessitam de avaliação clínica padrão e de ferramentas diagnósticas adicionais 21 É de fundamental importância a abordagem do tema, em virtude de suas prováveis e graves repercussões quando ocorre atraso na sua identificação (em geral, 47\%). A consequência mais importante do atraso diagnóstico e da conduta cirúrgica, é a perfuração apendicular, 22 com proporção em cerca de $25 \%$, observada em relatos da literatura mundial, 16 e a mais grave, o óbito da puérpera. $4-7,12$

O aumento de duas a três vezes na presença de perfuração do apêndice e peritonite nas puérperas, podendo resultar grave morbidade e/ou mortalidade materna, ressaltam a importância da divulgação da frequência de casos de apendicite aguda em puérperas entre clínicos e obstetras. É de suma importância que estejam atentos em relação a sua ocorrência, e assim possam realizar o diagnóstico o mais precocemente possível, visando a redução do número de complicações e da mortalidade destas pacientes. 4

\section{CONFLITOS DE INTERESSE}

Os autores declaram inexistência de conflitos de interesse na realização deste trabalho. 


\section{REFERÊNCIAS}

1. Augustin G, Majerovic M. Non-obstetrical acute abdome during pregnancy. Eur J Obstet Gynecol Reprod Biol. 2007: 131 (1): 4-12.

2. Gadelha OS, Costa AG, Câmara Filha EL, Buriti FMS, Fernandes AKS. Abdome agudo não obstétrico durante a gravidez: aspectos diagnósticos e manejo. Femina. 2009; 37 (3): 123-9.

3. Sharp HT. The acute abdome during pregnancy. Clin Obstet Gynecol 2002: 45 (2): 405-13.

4. Guttman R, Goldman RD, Koren G. Appendicitis during pregnancy. Can Fam Physician. 2004; 50 (3): $355-7$.

5. Bickell NA, Aufses Jr AH, Rojas M, Bodian C. How times affects the risks of rupture in appendicitis. J Am Coll Surg. 2006; 202 (3): 401-6.

6. Dalaqua M, Corsi PR. Apendicite aguda na gestação. Arq Med Hosp Fac Cienc Santa Casa São Paulo. 2006; 51 (1): 4-9.

7. Figueiredo FAZ, Corso CO. Apendicectomia laparoscópica na gestante. Rev Col Bras Cir 2002; 29 (5): 284-7.

8. Young BC, Hamar BD, Levine D, Roqué H. Medical management of ruptured appendicitis in pregnancy. Obstet Gynecol. 2009; 114 (2): 453-6.

9. Serruya SJ, Cecatti JG, Lago TG. O Programa de Humanização no Pré-natal e Nascimento do Ministério da Saúde no Brasil: resultados iniciais. Cad Saúde Pública .2004; 20 (5): 1281-9.

10. Santos AM. Abordagem social do conhecimento. Lat Am J Develop. 2020; 4 (2): 129-35.

11. Panisset KSP, Santos Jr JDO, Pereira BB, Cunha AJJA. Sazonalidade dos nascimentos no Estado do Rio de Janeiro: 1995-2005. Lat Am J Develop.2021: 6 (2): 671-88.

12. Torres OJM, Lins ALL, Nunes PMS, Corrêa FCF, Carvalho Jr OS, Castro FC. Avaliação ultrasonográfica da apendicite aguda. Rev Col Bras Cir. 2001; 28 (1): 39-43.

13. Chung $\mathrm{CH}, \mathrm{Ng} \mathrm{CP}$, Lai KK. Delays by patients, emergency physicians, and surgeons in the management of acute appendicitis: retrospective study. Hong Kong Med J. 2000; 6 (3): 254-9.

14. Tundidor-Bermudez AM, Diégues JAA, Montes-de-Oca- Mastrapa JI. Manifestaciones urológicas de la apendicitis aguda. Arch Esp Urol 2005; 58 (3): 207-12.

15. Puskar D, Bedalov G, Fridrih S, Vuckovic I, Banek T, Pasini J. Urinalysis, ultrasound analysis, and renal dynamic scintigraphy in acute appendicitis. Urology. 1995; 459 (1): 108-12.

16. Franca Neto AH, Amorim MMR, Nóbrega BMSV. Apendicite aguda na gravidez. Revisão de literatura. Rev Assoc Med Bras. 2015; 61 (2): 170-0. 
17. Barloon TJ, Brown BP, Abu-Yousef MM, Warnouck N, Berbaum KS. Sonography of acute appendicitis in pregnancy. Abdom Imaging. 1995; 20 (2): 149-51.

18. Hurwitz LM, Yoshizumi T, Reiman RE, Goodman PC, Paulson EK, Frush $\mathrm{DP}$, et al. Radiation dose to the fetus from body MDCT during early gestation. A J R Am J Roentgenol. 2006; 186 (3): 871-6.

19. Yilmaz HG, Akgun Y, Bac B, Celik Y. Acute appendicitis in pregnancy-risk factors associated with principal outcomes: a case control study. Int J Surg. 2007; 5 (3): 192-7.

20. Gaitán HG, Revetz L, Farquhar C. Laparoscopy for the management of acute lower abdominal pain in women of childbearing age. Cochrane Database Syst Rev. 2011 Jan 19;(1):CD007683

21. Kozan R, Bayhan H, Soykan Y, Anadol AZ, Sare M, Aytac AB. Acute Appendicitis: How to Manage?. Sisli Etfal Hastan Tip Bul. 2020; 11: 54 (4): 457-62.

22. Di Saverio S, Podda M, Simone B, Ceresoli M, Augustin G, Gon A, Boermeester M et al. Diagnosis and treatment of acute appendicitis. W J Emerg Surg. 2020: 15 (1): 27-42. 